\title{
Multimodal Multi-tasking for Skin Lesion Classification Using Deep Neural Networks
}

\author{
Rafaela Carvalho ${ }^{1,2(\bowtie)}\left(\mathbb{D}\right.$, João Pedrosa $^{2,3}\left(\mathbb{C}\right.$, and Tudor Nedelcu ${ }^{1}(\mathbb{C}$ \\ 1 Fraunhofer Portugal AICOS, Rua Alfredo Allen 455, 4200-135 Porto, Portugal \\ $\{$ rafaela.carvalho, tudor.nedelcu $\}$ fraunhof er.pt \\ 2 Faculty of Engineering of the University of Porto, Rua Dr. Roberto Frias, \\ 4200-465 Porto, Portugal \\ 3 Institute for Systems and Computer Engineering, Technology and Science \\ (INESC TEC), Rua Dr. Roberto Frias, 4200-465 Porto, Portugal \\ joao.m.pedrosa@inesctec.pt
}

\begin{abstract}
Skin cancer is one of the most common types of cancer and, with its increasing incidence, accurate early diagnosis is crucial to improve prognosis of patients. In the process of visual inspection, dermatologists follow specific dermoscopic algorithms and identify important features to provide a diagnosis. This process can be automated as such characteristics can be extracted by computer vision techniques. Although deep neural networks can extract useful features from digital images for skin lesion classification, performance can be improved by providing additional information. The extracted pseudo-features can be used as input (multimodal) or output (multi-tasking) to train a robust deep learning model. This work investigates the multimodal and multi-tasking techniques for more efficient training, given the single optimization of several related tasks in the latter, and generation of better diagnosis predictions. Additionally, the role of lesion segmentation is also studied. Results show that multi-tasking improves learning of beneficial features which lead to better predictions, and pseudo-features inspired by the ABCD rule provide readily available helpful information about the skin lesion.
\end{abstract}

Keywords: Skin lesions $\cdot$ Multimodal learning $\cdot$ Multi-task learning

\section{Introduction}

Skin cancer refers to the abnormal growth of aberrant skin cells and is divided into two main types: malignant melanoma and non-melanoma skin cancer, which are ranked the 19th and 4th most common cancers in the world [16], respectively.

Early diagnosis improves prognosis of skin cancer. If it happens in a localized stage, patients have a $98 \%$ 5-year relative survival rate, whereas if diagnosed in a distant stage, it drops to $17 \%$ [14]. The lives of human beings highly depend on a timely diagnosis, however this process can be challenging due to the variability in the appearance of skin lesions. This has led to the development of a non-invasive

(C) The Author(s) 2021

G. Bebis et al. (Eds.): ISVC 2021, LNCS 13017, pp. 27-38, 2021.

https://doi.org/10.1007/978-3-030-90439-5_3 
imaging technique: dermoscopy, which provides a higher diagnosis accuracy by revealing dimensions of skin morphological characteristics imperceptible to the naked eye. The most commonly employed algorithms for lesion inspection using dermoscopy are: ABCD rule of dermoscopy, Menzies scoring method, and sevenpoint checklist [7]. Such algorithms allow for an increased performance of the diagnosis process but the process remains highly dependent on the observer's experience and training. Moreover, as dermatology is suited for telemedicine and with the burden in healthcare systems at present, automated systems may be the answer towards diagnosis of malignant skin lesions at an early stage.

The emergence of deep learning has promoted the development of promising skin lesion analysis methodologies. The majority of the works found in the literature use transfer learning from well-established convolutional neural networks (CNNs) pre-trained on the ImageNet dataset [12].

One of the most important implementations of skin lesion classification with CNNs was achieved by Esteva et al. (2017) [4]. The authors adapted the pretrained Inception v3 [17] network for skin lesion diagnosis and trained it on a large private dataset of 129.450 clinical images, consisting of 2032 different diseases. For data balancing, a hierarchical partitioning algorithm using a taxonomy tree was used. They reported performance results on par with 21 experts, indicating a solution with a level of competence comparable to dermatologists.

Multi-task learning (MTL) has been gaining traction and Liao et al. (2018) [10] built a deep MTL framework to exploit the correlation between skin lesions and their body site distributions. The dermatology images used in the study were collected from DermQuest (deactivated in 2019), an atlas with 25 types of skin lesions and 23 different body location labels.

Multimodal methods have also been proposed for skin lesion diagnosis. Yap et al. (2018) [19] investigated the combination of available data for classification. The ResNet-50 [5] with weights pre-trained on ImageNet was used to reduce the overfitting for a dataset of 2917 cases containing both clinical and dermoscopic images. When trained on dermoscopic images, it presented higher accuracy than on clinical images. Nonetheless, the combination of feature information from dermoscopic and clinical images outperformed single modal CNN, which indicates that both imaging modalities have dissimilar classification information.

Kawahara et al. (2019) [8] proposed a multimodal multi-task deep CNN with a base model Inception v3, pre-trained on ImageNet. The architecture was trained on multimodal data (clinical and dermoscopic images, as well as patient metadata), to classify the 7-point checklist criteria and perform skin lesion diagnosis. Their dataset containing the 2022 images and metadata has been made publicly available online. The network was trained using a combination of multimodal and multi-tasking losses, thus creating a robust model.

The focus of this work is to implement a system for skin lesion diagnosis using deep neural networks, while investigating the emerging multi-tasking and multimodal methods for more efficient training and an enhanced prediction. ABCD rule inspired pseudo-features are extracted through computer vision techniques and used as output (multi-tasking) or input (multimodal) to train a robust deep learning model. 


\section{Methodology}

\subsection{Dataset}

The dataset employed in this work is from the ISIC 2017 challenge [3] and contains dermoscopic images which are labelled, according to expert consensus and pathology report information, as malignant melanocytic melanoma (MM), benign non-melanocytic seborrheic keratosis (SK) and benign melanocytic nevus $(\mathrm{NV})$. Expert manual tracing of the lesion boundaries and superpixel-mapped annotations of the presence of dermoscopic criteria: pigment network $(\mathrm{PN})$, negative network (NN), milia like cyst (MLC) and streaks (ST), are also provided.

We used the data split set by the challenge: a training set with 2000 images (1372 NV, 374 MM, $254 \mathrm{SK}$ ), 150 validation (78 NV, $30 \mathrm{MM}, 42 \mathrm{SK}$ ) and 600 test samples (393 NV, 117 MM, $90 \mathrm{SK}$ ). By using the same distribution, this work's results can be compared with other methods from the literature, in particular the ISIC 2017 challenge submissions.

\subsection{ABCD Rule Feature Extraction}

Among the diagnosis algorithms of dermatology, the most popular and responsible for inspiring many CAD systems is the ABCD rule of dermoscopy [7]. According to this method, skin lesions can be characterized based on four criteria: asymmetry, border, color and number of dermoscopic structures.

As the dataset of this work does not contain expert annotations of ABCD criteria, extraction of those traits and subsequent data labelling is required for application of the multimodal and multi-tasking techniques. The framework for asymmetry and border extraction can be seen in Fig. 1. Color was not investigated in this paper and is under consideration for future work.

Asymmetry. A skin lesion is asymmetric when its two halves do not match. The asymmetry score $\left(A_{\text {score }}\right)$ of a lesion is important when evaluating its malignant potential. This assessment will be concerning the shape: benign lesions are usually circular, whereas asymmetric lesions provide a warning sign of MM.

The major axis of the lesion is identified using the provided segmentation mask by calculating its orientation $(\theta)$, as described by Celebi et al. (2007) [2]. The lesion is rotated $\theta$ degrees clockwise to align its major and minor axes with the $\mathrm{x}$ and $\mathrm{y}$ axes of the image, and is centered. For each axis, the mirrored version of one half is overlapped with its correspondent and their exclusive OR area is computed. A non-overlapping area mask is obtained, allowing to estimate an asymmetry ratio between the preceding and the total lesion area.

According to the ABCD rule, skin lesions are divided in three levels: fully symmetric (0), asymmetric on one (1) and two axes (2). For the definition of such classes given the asymmetry ratio, a threshold is set in such a way that more than half of MMs have $A_{\text {score }}=2$ and approximately $60 \%$ of benign lesions are scored 0 or 1 (please note that MMs are typically asymmetric whereas both halves of benign lesions usually match). Hence, if the non-overlapping area exceeds $6 \%$ of the lesion area, the lesion is considered asymmetric in that axis. 
Border. MMs are usually associated with irregular and poorly defined borders, while NV's are even and smooth. In the clinical evaluation of the border, the sharpness of the transition from the lesion to the skin $\left(B_{\text {score }}\right)$ is determined.

The gradient is computed along the border points, using the blue channel as skin lesions are usually more noticeable in this channel [1]. Firstly, the contour is characterized with 200 equidistant points and the normal direction of each point is found. The gradient in each border area is reduced to the mean difference of the pixel intensities along a line, whose length equals $30 \%$ of the lesion radius. The lesion is subsequently divided into 8 equi-angle slices, and, for each, an average value of the gradient is computed, as in Iyatomi et al. (2007) [6].

For definition of the $B_{\text {score }}$, we set an empiric threshold value of 50 for the average gradient which classifies the transition in each particular slice into either soft (0) or abrupt (1). This procedure is executed for all the divisions, resulting in a final $B_{\text {score }}$ between 0 and 8 .

Dermoscopic Structures. The superpixel annotations from the dataset are used to infer the existence of PN, NN, MLC and ST structures and, consequently, label the data according to their presence or absence.

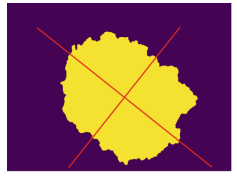

(a) Original mask

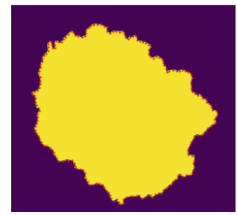

(f) Equidistant contour points

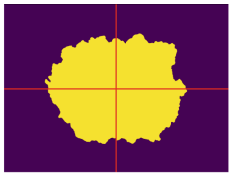

(b) Rotated and centered

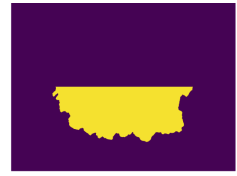

(c) Top half mirrored

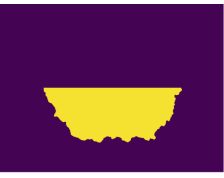

(d) Bottom half

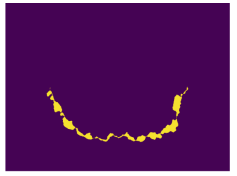

(e) Exclusive OR area

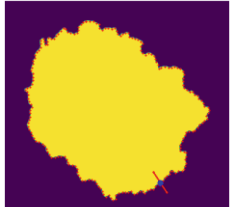

(g) Normal direction at point

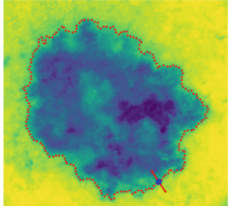

(h) Normal periphery at point

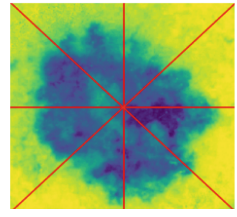

(i) Equi-angle slices

Fig. 1. Illustration of asymmetry (top row) and border (bottom row) extraction.

\subsection{Proposed Model}

EfficientNet [18] is a group of CNNs developed based on network scaling (depth, width and resolution), whose dimensions vary depending on the variant selected (B0 to B7). This architecture focuses on accuracy and efficiency, and is able to achieve state-of-the-art results while being multiple times smaller and faster [18].

An EfficientNet-B3 architecture, with weights and biases pre-trained on ImageNet, is used as the backbone of the experiments performed in this work. Variant 
B3 is chosen because of the balance when comparing the number of parameters required and accuracy achieved with other CNNs.

Each dermoscopic image is resized to $(300 \times 300)$ pixels to make it compatible with the original dimensions of the network. Data augmentation transformations to simulate new samples and avoid overfitting are applied: rotations in the range of $[1,5]$ degrees, zoom between $80 \%$ and $120 \%$, horizontal flips and shifts up to $20 \%$ of the image size, and brightness adjustment in the range $[0.2,0.8]$.

To accommodate the network for the desired tasks, a global average pooling layer is introduced on top of the frozen base network to reduce the number of parameters for the classifier, followed by batch normalization and dropout (rate of 0.2 ) layers as regularization to prevent overfitting. The original classifier is replaced by a softmax layer with 3 (skin lesion classes) neurons and parameters are updated using a learning rate of $1 \mathrm{e}-3$. A frozen layer approach [8] is adopted to improve training by reducing the effect of transfer learning for the bottom layers, which are designed to extract more general features. The approach consists of unfreezing a EfficientNet-B3 block and fitting the model for 5 epochs using a lower learning rate $(1 \mathrm{e}-6)$, repeating this until all blocks are unfrozen.

Each CNN model is compiled using the Adam optimization algorithm [9] and the loss function is specified to be categorical cross entropy (CCE).

\subsection{Class Balancing Techniques}

The skin lesion dataset of this work is imbalanced between classes. As a result, bias is introduced towards the most represented class, compromising the performance of the models.

A procedure which ensures data frequency [8] is carried out, i.e., each batch is verified to always hold at least 1 positive sample of every unique skin lesion and dermoscopic structure class, resulting in a batch size of 6 . Since the category labels are not mutually exclusive, it is important that the same sample is not represented twice in the same batch; this is ensured by removing each case from the set after picking it. By guaranteeing one case of each unique label, model weights will be updated based on all the unique labels in each gradient descent step [8]. Nonetheless, while this improves class balance, there is still imbalance as including a case within one category will also include its labels in all other categories. To further address this issue, class weights are introduced. These are used to penalize the misclassification of samples from the minority classes. The weight $W_{i}$ of each class $i$ is given by $W_{i}=\frac{N}{C \cdot n_{i}}$, where $N$ is the total number of samples, $C$ is the number of classes, and $n_{i}$ is the number of samples for class $i$.

By applying this modification $\left(W_{i}\right)$ in the loss function employed in this work (CCE), a weighted loss function (Eq. 1) is obtained:

$$
\text { Weighted Cross Entropy }=-\sum_{i=1}^{K} W_{i} \cdot y_{i} \cdot \log \left(\hat{y}_{i}\right)
$$

with $K$ equaling to the number of scalar values in the model output, $\hat{y}_{i}$ being the $i$-th predicted value and $y_{i}$ the corresponding true value. 


\section{$3 \quad$ Experiments}

Hand-crafted features inspired by the ABCD rule are extracted and used as new auxiliary tasks to be predicted or as additional input merged with the embeddings extracted by the CNN. Additionally, since the ISIC 2017 dataset contains segmentation masks and extensive work was conducted regarding this aspect, the impact of segmentation maps is also investigated in this study.

\subsection{ABCD Rule for Multimodal Multi-tasking}

This dataset does not provide access to expert annotations of dermoscopic criteria (except dermoscopic structures), unlike the 7-point checklist dataset [8]. Thus, hand-crafted pseudo-features based on the ABCD rule are extracted by computer vision techniques, and are used as input (multimodal) or output (multitasking) to train a robust model.

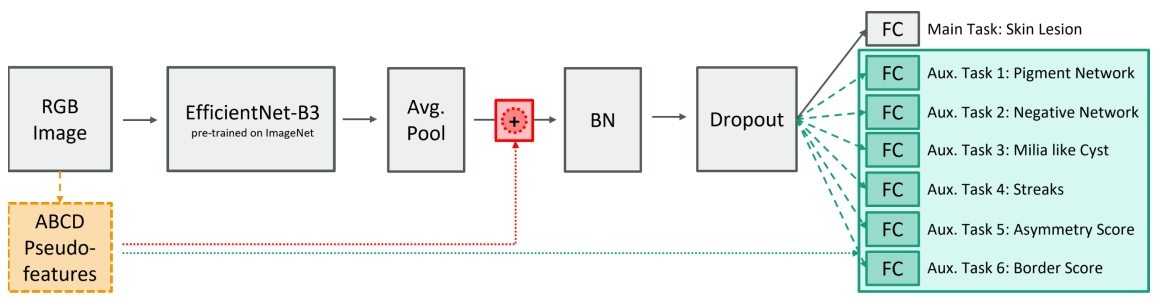

Fig. 2. Diagram of the models' structure. Baseline model is represented in grey $\square$; modifications regarding the multi-tasking and multimodal approaches are presented in green $\square$ and red $\square$, respectively. (Color figure online)

Multi-tasking. Multi-tasking is performed to guide the model to extract meaningful features for a specific task by predicting other closely related auxiliary tasks. The aim of this experiment is detecting ABCD traits to infer a skin lesion diagnosis. The motivation behind this design is to make use of the correlation between these criteria and lesion diagnosis: for example, MM has typically high $A_{\text {score }}$ and $B_{\text {score }}$; in terms of dermoscopic structures, milia like cyst is usually indicative of SK [15] whereas negative network is a MM-specific structure [11].

The baseline model is adapted to a multi-task network with a hard parameter sharing approach, i.e., sharing the hidden layers between all tasks while keeping separate task-specific output softmax layers (Fig. 2). This model is able to simultaneously predict the skin lesion diagnosis and classify ABCD criteria. By predicting different characteristics of the lesion, these helpful representations are shared with the main task, thus improving diagnosis. All multi-task models studied focus on the classification of the presence of dermoscopic structures - $\mathrm{ABC}(\mathrm{D})$. However, prediction of $A_{\text {score }}$ and $B_{\text {score }}$ is tested concurrently $(\mathrm{AB}) \mathrm{C}(\mathrm{D})$ - or each separately - $(\mathrm{A}) \mathrm{BC}(\mathrm{D})$ and $\mathrm{A}(\mathrm{B}) \mathrm{C}(\mathrm{D})$. 
Multimodal Multi-tasking. Through this approach, the network is provided with readily available information tailored to what physicians use ( $\mathrm{BCD}$ rule) and which are thus characteristics proven to be relevant for malignancy classification. With these appropriate features, we aim to decrease the amount of overfitting, therefore enhancing the performance of CNN-based algorithms.

Late feature fusion is performed with the multi-task model set to receive two inputs, dermoscopic images and two characteristics of skin lesions that doctors look for when diagnosing MMs: asymmetry and border. Hence, the aforementioned hand-crafted ABCD features are directly concatenated with the feature vector obtained by the EfficientNet-B3, as observed in Fig. 2. Not all features might be beneficial to the classification task, some can possibly weaken the performance of the classifier. Consequently, different cases are tested: including only asymmetry or border information - (A)BC(D) and $\mathrm{A}(\mathrm{B}) \mathrm{C}(\mathrm{D})$ multimodal multi-task - or both simultaneously - $(\mathrm{AB}) \mathrm{C}(\mathrm{D})$ multimodal multi-task.

\subsection{Role of Segmentation in Lesion Classification}

Artifacts such as hair, ruler markings, non-target lesions, and background noise in images can potentially deceive a model's classifier. A segmentation step is investigated to verify whether removing the pixel intensities outside the target lesion is advantageous for classification. Additionally, we test calculation of dynamic weights, i.e., weights based on the distribution of classes in each batch.

The performances of the $\mathrm{ABC}(\mathrm{D})$ multi-task model with dynamic weights receiving different inputs, observed in Fig. 3, are assessed.

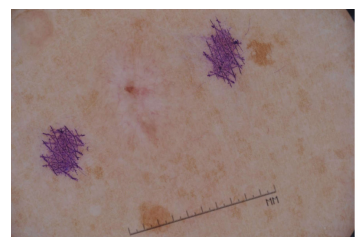

(a) Original Image

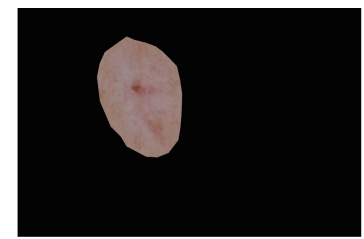

(b) Segmented Image

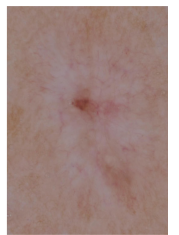

(c) Cropped Image

Fig. 3. Examples of the inputs for the multi-task model.

\section{Results and Discussion}

To evaluate the performance of the techniques tested in this work, the area under the curve (AUC), sensitivity (SE), specificity (SP), and balanced multiclass accuracy (BMA) are reported. Table 1 summarizes the overall diagnosis results and Table 2 presents the AUC for dermoscopic features classification. Bold values highlight the best result for each metric. 
Table 1. Results of the models for the diagnosis task.

\begin{tabular}{|c|c|c|c|c|c|c|c|c|c|c|}
\hline \multirow{2}{*}{ Model } & \multicolumn{3}{|c|}{ AUC } & \multicolumn{3}{|c|}{$\mathrm{SE}(\%)$} & \multicolumn{3}{|c|}{ SP (\%) } & \multirow{2}{*}{ BMA (\%) } \\
\hline & NV & MM & SK & NV & MM & SK & NV & MM & SK & \\
\hline Baseline model & 0,84 & 0,81 & 0,90 & 84 & 42 & 68 & 65 & 93 & 90 & 65 \\
\hline $\mathrm{ABC}(\mathrm{D})$ multi-task & 0,82 & 0,74 & 0,88 & 69 & 50 & 80 & 81 & 84 & 84 & 66 \\
\hline (A)BC(D) multi-task & 0,82 & 0,71 & 0,89 & 58 & 46 & 87 & 90 & 84 & 73 & 64 \\
\hline $\mathrm{A}(\mathrm{B}) \mathrm{C}(\mathrm{D})$ multi-task & 0,81 & 0,67 & 0,88 & 62 & 29 & 90 & 84 & 90 & 69 & 60 \\
\hline$(\mathrm{AB}) \mathrm{C}(\mathrm{D})$ multi-task & 0,81 & 0,69 & 0,87 & 57 & 32 & 88 & 84 & 88 & 67 & 59 \\
\hline (A)BC(D) multimodal multi-task & 0,82 & 0,75 & 0,89 & 63 & 38 & 84 & 83 & 89 & 73 & 62 \\
\hline $\mathrm{A}(\mathrm{B}) \mathrm{C}(\mathrm{D})$ multimodal multi-task & 0,83 & 0,73 & 0,90 & 66 & 36 & 87 & 84 & 91 & 72 & 63 \\
\hline$(\mathrm{AB}) \mathrm{C}(\mathrm{D})$ multimodal multi-task & 0,83 & 0,74 & 0,90 & 53 & 44 & 90 & 91 & 83 & 69 & 62 \\
\hline
\end{tabular}

Table 2. Area under curve (AUC) values of the models for the auxiliary tasks. For asymmetry (Asym) and border (Bord), the average AUC is considered.

\begin{tabular}{lcccccc}
\hline \multirow{2}{*}{ Model } & \multicolumn{7}{c}{ AUC } \\
& PN & NN & MLC & ST & ASYM & BorD \\
\hline ABC(D) multi-task & 0,76 & 0,61 & 0,61 & 0,77 & - & - \\
(A)BC(D) multi-task & $\mathbf{0 , 7 9}$ & 0,66 & 0,63 & 0,84 & $\mathbf{0 , 5 6 7}$ & - \\
A(B)C(D) multi-task & $\mathbf{0 , 7 9}$ & 0,67 & 0,59 & 0,81 & - & 0,822 \\
(AB)C(D) multi-task & 0,78 & 0,65 & 0,58 & 0,80 & 0,563 & $\mathbf{0 , 8 2 4}$ \\
(A)BC(D) multimodal multi-task & $\mathbf{0 , 7 9}$ & 0,69 & 0,63 & 0,84 & - & - \\
A(B)C(D) multimodal multi-task & 0,78 & $\mathbf{0 , 7 0}$ & $\mathbf{0 , 6 4}$ & $\mathbf{0 , 8 7}$ & - & - \\
(AB)C(D) multimodal multi-task & $\mathbf{0 , 7 9}$ & $\mathbf{0 , 7 0}$ & 0,61 & 0,83 & - & - \\
\hline
\end{tabular}

The performances of the networks are similar regarding the BMA (Table 1). The strong correlation between dermoscopic features and MM is observed in this experiment, when dermatological attributes are used as auxiliary tasks (ABC(D) multi-task), as denoted by the $8 \%$ increase in MM SE and $7 \%$ decrease in SP. Other experiments regarding prediction of more tasks did not improve the BMA. With $A_{\text {score }}$ prediction as an additional task, SP for NV is increased by $9 \%$ while $\mathrm{SE}$ is reduced by $11 \%$. The opposite behaviour is observed for prediction of SK, where SE is increased by $10 \%$ while SP is reduced by $15 \%$. When predicting all auxiliary tasks related to asymmetry, border and dermoscopic features, the model achieves the lowest performance regarding BMA. This reduction could be induced by the balancing techniques, which are difficult to perform for a small dataset with several outputs.

To investigate if a multimodal multi-tasking approach can boost the classifier performance, the combination of the ABCD rule inspired pseudo-features and CNN features was tested. No major improvement regarding the prediction of skin lesion diagnosis is observed, confirming that the network is able to automatically learn good image representations. However, AUC values of the multimodal multi-tasking models are slightly enhanced when compared to its 
corresponding backbone ( $\mathrm{ABC}(\mathrm{D})$ multi-task): there is an improvement for $\mathrm{NV}$ and SK when using the border gradient and the addition of asymmetry benefits MM. Regarding dermoscopic structures, we noticed an improvement in their detection (Table 2), specifically with the introduction of the border gradient.

The GradCAM technique [13] was applied to have visual feedback on where the network's activations, previous to the softmax layer, were more predominant. Analyzing Fig. 4, the superiority of multi-tasking is confirmed, by showing that closely related auxiliary tasks help the network to focus on the lesion.

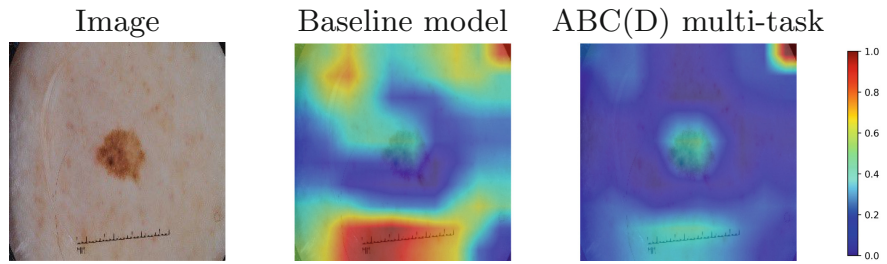

Fig. 4. GradCAM diagnosis heat maps from the baseline and multi-task models. Color represents the degree of activation from low (blue - 0) to high (red - 1). (Color figure online)

Table 3 depicts the role of lesion segmentation in the classifier's performance.

Using a segmentation mask to remove background information and pass only the lesion as input to the model considerably decreases performance, when comparing it with the unaltered input. Segmented images possibly removed contextual information which could be relevant for the classification task. Results of the original dataset and the bounding box images are extremely similar, with the original images providing advantage in the AUC of MM, but the cropped images achieving higher MM SE, which could be preferable in a clinical setting.

Table 3. Results of models with modified images as inputs.

\begin{tabular}{lcccccccccc}
\hline \multirow{2}{*}{ Model input } & \multicolumn{3}{c}{ AUC } & \multicolumn{4}{c}{ SE (\%) } & \multicolumn{4}{c}{ SP (\%) } & \multirow{2}{*}{ BMA (\%) } \\
& NV & MM & SK & NV & MM & SK & NV & MM & SK & \\
\hline Original images & $\mathbf{0 , 8 4}$ & $\mathbf{0 , 7 5}$ & 0,89 & $\mathbf{7 6}$ & 45 & $\mathbf{7 8}$ & 80 & $\mathbf{8 9}$ & 84 & $\mathbf{6 6}$ \\
Segmented images & 0,75 & 0,69 & 0,82 & 75 & 44 & 50 & 62 & 82 & $\mathbf{9 1}$ & 56 \\
Cropped images & $\mathbf{0 , 8 4}$ & 0,73 & $\mathbf{0 , 9 1}$ & 67 & $\mathbf{5 2}$ & $\mathbf{7 8}$ & $\mathbf{8 2}$ & 78 & 88 & $\mathbf{6 6}$ \\
\hline
\end{tabular}

GradCAM was once again employed to compare the impact of segmentation maps on the network's activations. The heat maps (Fig. 5) suggest the network learns to roughly detect the skin lesion. Artifacts such as ruler markings are also activated, which was expected. For the diagnosis task, it is noticeable that the network trained on segmented images considers a wider portion of the image than 


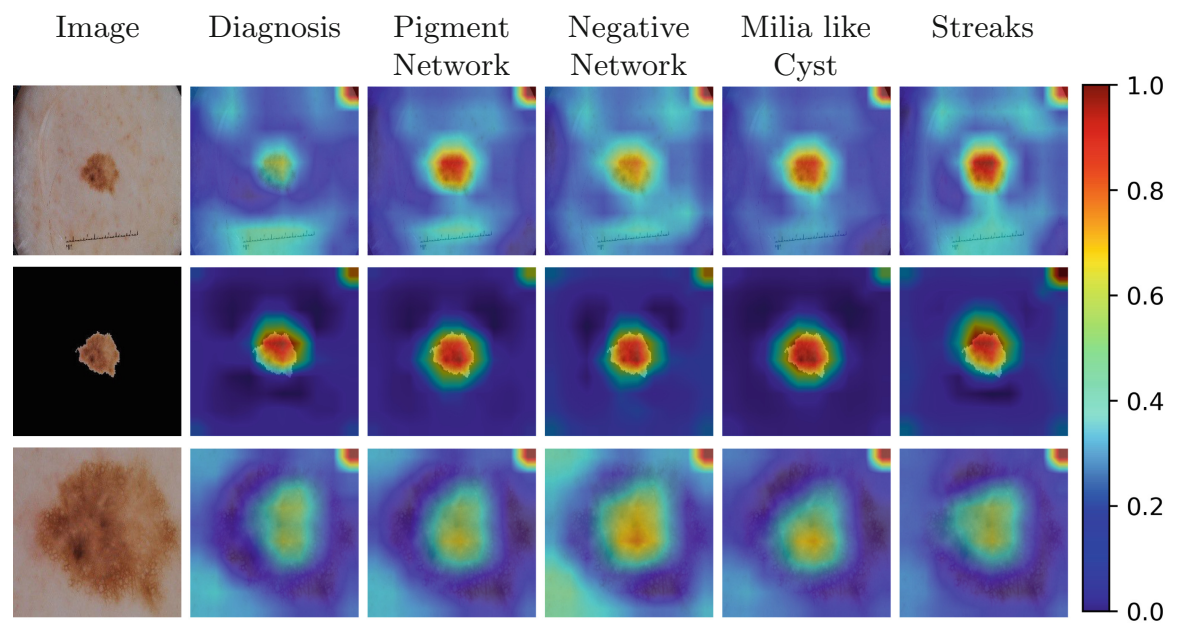

Fig. 5. Example of the different inputs for a skin lesion and respective GradCAM heat maps for each model task.

the original images, including parts for which there is no information, therefore leading to worse performance; activation on cropped images misses parts of the lesion which may contain important features to discriminate between classes. It is worth noting neighbor pixels surrounding the lesion are important as the difference between background (skin) and foreground (lesion) intensities provides relevant information regarding color and texture variations. The same behaviour is observed in the tasks related to dermoscopic criteria classification.

The leading approach of this work $(\mathrm{ABC}(\mathrm{D})$ multi-task) reached an average AUC of $87 \%$ for the diagnosis task, placing it among the top 35\% of the ISIC 2017 challenge leaderboard ${ }^{1}$. The most successful challenge submissions implemented ensembles of DL networks and extended the dataset with additional data sources [3], whereas this work was limited to a single model and no external data.

\section{Conclusion}

This paper proposes multi-tasking and multimodal strategies to improve stateof-the-art automated systems for the problem of skin lesion diagnosis, while handling the imbalanced dataset. To the best of our knowledge, there is a small number of comprehensive studies in the literature related to this topic, therefore the aim of the work is to contribute to this line of research.

The yielded results show potential in the use of MTL, proving that it allows different tasks to share meaningful features and beneficial representations. This was corroborated by the heat maps obtained for the baseline and $\mathrm{ABC}(\mathrm{D})$ multitask models. Auxiliary classification of ABCD rule criteria did not translate

\footnotetext{
${ }^{1}$ https://challenge.isic-archive.com/leaderboards/2017.
} 
into enhanced performance, which can possibly be explained by the difficulty to train a model which predicts a large number of tasks on a small and imbalanced dataset. Plus, this information was manually extracted, divided in classes and labelled, thus being imperfect and introducing bias. The use of ABCD ratings annotated by experts has the potential to yield superior results. Even so, the multimodal technique with such descriptors increased AUCs.

Additionally, this work provided insight on the role of background information, through the comparison of the same model with different inputs: original dermoscopic view, images cropped around the lesion and images containing only lesion information. The analysis of the heat maps for diagnosis and dermoscopic criteria classification tasks in models with a segmentation step confirmed that all networks roughly detected the lesion, but the latter led to poor results, explained by the removal of contextual information.

The learning paradigms investigated in this work are active areas for improvement and can lead to reliable skin lesion classification systems. Multi-tasking models provide the advantage of running on inference without extracting ABCD features. Moreover, multimodal and multi-tasking can potentially enhance the possibility of explainability. Further future work considers developing the models presented to incorporate patient information and/or clinical images as additional inputs, since combining complementary information from multiple modalities has the potential to improve performance. Given the limitation of the small size of the dataset, these approaches must be tested on a larger one.

Acknowledgments. This work was done under the scope of project "DERM.AI: Usage of Artificial Intelligence to Power Teledermatological Screening", and supported by national funds through 'FCT-Foundation for Science and Technology, I.P.', with reference DSAIPA/AI/0031/2018.

\section{References}

1. Celebi, M.E., Iyatomi, H., Schaefer, G., Stoecker, W.V.: Lesion border detection in dermoscopy images. Comput. Med. Imaging Graph. 33(2), 148-153 (2009)

2. Celebi, M.E., et al.: A methodological approach to the classification of dermoscopy images. Comput. Med. Imaging Graph. 31(6), 362-373 (2007)

3. Codella, N.C., et al.: Skin lesion analysis toward melanoma detection: a challenge at the 2017 international symposium on biomedical imaging (isbi), hosted by the international skin imaging collaboration (isic). In: 2018 IEEE 15th International Symposium on Biomedical Imaging (ISBI 2018), pp. 168-172. IEEE (2018)

4. Esteva, A., et al.: Dermatologist-level classification of skin cancer with deep neural networks. Nature 542(7639), 115-118 (2017)

5. He, K., Zhang, X., Ren, S., Sun, J.: Deep residual learning for image recognition. In: Proceedings of the IEEE Conference on Computer Vision and Pattern Recognition, pp. 770-778 (2016)

6. Iyatomi, H., Oka, H., Celebi, M.E., Tanaka, M., Ogawa, K.: Parameterization of dermoscopic findings for the internet-based melanoma screening system. In: 2007 IEEE Symposium on Computational Intelligence in Image and Signal Processing, pp. 189-193. IEEE (2007) 
7. Johr, R.H.: Dermoscopy: alternative melanocytic algorithms-the abcd rule of dermatoscopy, menzies scoring method, and 7-point checklist. Clin. Dermatol. 20(3), 240-247 (2002)

8. Kawahara, J., Daneshvar, S., Argenziano, G., Hamarneh, G.: Seven-point checklist and skin lesion classification using multitask multimodal neural nets. IEEE J. Biomed. Health Inform. 23(2), 538-546 (2018)

9. Kingma, D.P., Ba, J.: Adam: A method for stochastic optimization. arXiv preprint arXiv:1412.6980 (2014)

10. Liao, H., Luo, J.: A deep multi-task learning approach to skin lesion classification. arXiv preprint arXiv:1812.03527 (2018)

11. Pizzichetta, M.A., et al.: Negative pigment network: an additional dermoscopic feature for the diagnosis of melanoma. J. Am. Acad. Dermatol. 68(4), 552-559 (2013)

12. Russakovsky, O., et al.: Imagenet large scale visual recognition challenge. Int. J. Comput. Vis. 115(3), 211-252 (2015)

13. Selvaraju, R.R., Cogswell, M., Das, A., Vedantam, R., Parikh, D., Batra, D.: Gradcam: Visual explanations from deep networks via gradient-based localization. In: Proceedings of the IEEE International Conference on Computer Vision, pp. 618$626(2017)$

14. Siegel, R.L., Miller, K.D., Jemal, A.: Cancer statistics, 2019. CA: Canc. J. Clin. 69(1), 7-34 (2019)

15. Stricklin, S., Stoecker, W., Oliviero, M., Rabinovitz, H., Mahajan, S.: Cloudy and starry milia-like cysts: how well do they distinguish seborrheic keratoses from malignant melanomas? J. Euro. Acad. Dermatol. Venereology 25(10), 1222-1224 (2011)

16. Sung, H., et al.: Global cancer statistics 2020: globocan estimates of incidence and mortality worldwide for 36 cancers in 185 countries. CA: Canc. J. Clin. 71(3), 209-249 (2021)

17. Szegedy, C., et al.: Going deeper with convolutions. In: Proceedings of the IEEE Conference on Computer Vision and Pattern Recognition, pp. 1-9 (2015)

18. Tan, M., Le, Q.: Efficientnet: rethinking model scaling for convolutional neural networks. In: International Conference on Machine Learning, pp. 6105-6114. PMLR (2019)

19. Yap, J., Yolland, W., Tschandl, P.: Multimodal skin lesion classification using deep learning. Exp. Dermatol. 27(11), 1261-1267 (2018)

Open Access This chapter is licensed under the terms of the Creative Commons Attribution 4.0 International License (http://creativecommons.org/licenses/by/4.0/), which permits use, sharing, adaptation, distribution and reproduction in any medium or format, as long as you give appropriate credit to the original author(s) and the source, provide a link to the Creative Commons licence and indicate if changes were made.

The images or other third party material in this chapter are included in the chapter's Creative Commons licence, unless indicated otherwise in a credit line to the material. If material is not included in the chapter's Creative Commons licence and your intended use is not permitted by statutory regulation or exceeds the permitted use, you will need to obtain permission directly from the copyright holder.

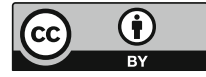

